\title{
Design of Optimum Microstrip to Substrate Integrated Waveguide Transition
}

\author{
Nassir Guellil ${ }^{1}$, Chemseddine Zebiri ${ }^{2,3}$, Djamel Sayad ${ }^{5}$,I.T.E. Elfergani ${ }^{3,4}$, Farid Djahli ${ }^{1}$, \\ Abdalfettah Asharaa ${ }^{3}$, Jonathan Rodriguez ${ }^{4}$, and Raed Abd-Alhameed ${ }^{3}$ \\ \{ i.t.e.elfergani@av.it.pt*\} \\ ${ }^{1}$ LIS laboratory, Department of Electronics, Ferhat Abbas University of Sétif 1, Sétif, Algeria, \\ guellilnassir@yahoo.fr, fdjahli@yahoo.fr \\ ${ }^{2}$ Department of Electronics, University of Ferhat Abbas, Sétif -1-, 19000 Sétif, Algeria; \\ czebiri@univ-setif.dz \\ ${ }^{3}$ Bradford-Renduchintala Centre for Space AI, University of Bradford, BD71DP, UK \\ ${ }^{4}$ Instituto de Telecomunicações, Campus Universitário de Santiago, Aveiro, Portugal \\ i.t.e.elfergani@av.it.pt \\ ${ }^{5}$ Department of Electrical Engineering, University of 20 Aout 1955-Skikda, 21000 Skikda, \\ Algeria
}

\begin{abstract}
The excitation of a Substrate Integrated Waveguide (SIW) makes use of costly and complex structures. In this paper, two simple structures have been presented to validate our computing results. This is a step discontinuity added to the microstrip line to produce an impedance matching between the SIW line and the line. Based on a small approximation of reflection, design equations are deduced, which allowed an optimal dimensioning of the step discontinuity $(\mathrm{L} \times \mathrm{W})$. This design strategy was tested by simulating several waveguides, and a good agreement between the optimal geometric parameters and the calculated values was obtained.
\end{abstract}

Keywords: SIW, transition, small reflection approximation.

\section{Introduction}

Substrate Integrated Waveguide is a new form of the waveguide. It is a mixed technology between the waveguide and printed circuits that benefit from the advantages and high performances of both technologies [1]. This new structure consists of a substrate metalized on up and down sides with two parallel rows of metallic vias inserted in the substrate. If the diameter of the metallic vias and the distance between them are chosen properly, the energy leaking between consecutive vias is negligible, hence, the structure behaves like a classical rectangular waveguide [2]. Because of the advantages of this technology most microwave components in a classical waveguide, such as filters, couplers, antenna and beam former, were modeled in SIW technology [3-7].

To interface a signal between microstrip technology and SIW structure a transition is used. Figure (1) presents the transition structure used in this work to connect a $50 \Omega$ microstrip line to the planar SIW. The integral design is realized on the same substrate. The purpose of the transition is to transform the quasi-TEM mode in the microstrip line to the dominant TE10 mode 
in the SIW. Since there is an impedance matching between the microstrip line and the SIW structure, return loss will be minimum, for this reason, the length and width of the transition structure has to be optimized.

This paper presents design equations for geometrical parameters (width and length) of the structure. These equations allow, in almost all cases, a direct calculation of the optimum width and length of the transition without a full-wave simulator. This method has been tested by applying it to microstrip to SIW transition with three examples using different substrates at different frequencies.

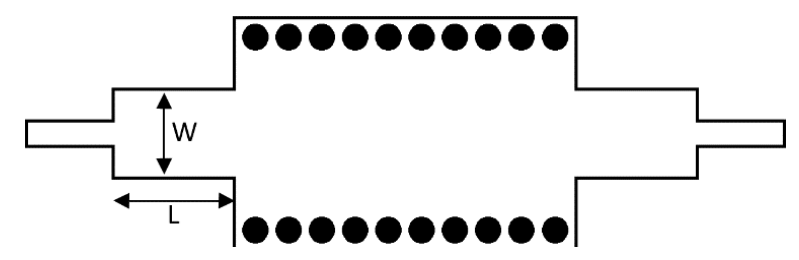

Fig. 1.Microstrip line to substrate integrated waveguide transition.

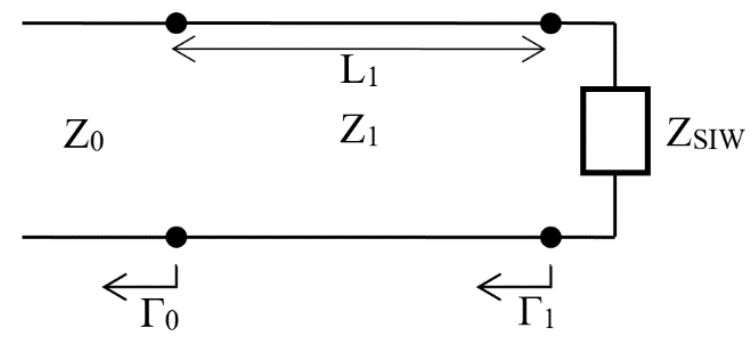

Fig. 2. Equivalent electric circuit.

\section{Design Procedure}

First, based on our previous work [7], a new formula for calculating the optimum width of a SIW, corresponding to the dominant mode, is used in this paper, by applying the Finite Difference Frequency Domain (FDFD) method. Secondly, the transition is a step discontinuity on a microstrip line as it is shown in Figure 1, where a microstrip line with a characteristics impedance $\mathrm{Z} 0=50 \mathrm{Ohm}$, connected to another microstrip line of impedance $\mathrm{Z} 1$ and length L1. The transition is responsible to realize the impedance matching between the impedance of the feeding line and the SIW impedance. So, to make a good compromise between impedances, the geometrical parameters W2 and L2 must be suitably chosen to get a lower return loss. Figure 2 shows a schematic of impedance matching considered in this work [8]. 
By using small reflection approximation, we can compute the total reflection Gamma seen by the feed line (Equation 1). We note that we ignore the mismatch at the end of the microstrip line where the quasi-TEM mode in the microstrip couples to theTE10 of the SIW [9].

$$
\Gamma=\Gamma_{0}+\Gamma_{1} e^{-2 j \theta}
$$

$$
\text { where } ; \Gamma_{0}=\frac{Z_{1}-Z_{0}}{Z_{1}+Z_{0}}, \Gamma_{1}=\frac{Z_{\text {siw }}-Z_{1}}{Z_{\text {siw }}+Z_{1}}, \theta=\frac{2 \pi}{\lambda_{g}} L_{1}
$$

In the $\mathrm{TE}_{10}$ propagation mode frequency region of interest, the SIW is equivalent to a conventional rectangular waveguide filled by a dielectric, hence, for the $\mathrm{TE}_{10}$ mode, the cross section impedance can be written as follows

$$
Z_{\text {siw }}=\frac{2 h}{W_{\text {eff }}} \frac{\eta_{0}}{\sqrt{\varepsilon_{r}}}\left[1-\left(\frac{f_{c}}{f}\right)^{2}\right]^{-\frac{1}{2}}
$$

We know that maximum power transfer from the input matching network to the waveguide structure will occur when:

$$
\frac{\partial^{2} \Gamma\left(Z_{1}, L_{1}\right)}{\partial Z_{1} \partial L_{1}}=0
$$

Solutions of (3) are:

$$
\left\{\begin{array}{c}
L_{1}=(k+1) \frac{\lambda_{g}}{2} \\
\quad \text { and } \\
Z_{1}=\frac{Z_{\text {siw }} \sqrt{Z_{o}}-Z_{o} \sqrt{Z_{\text {siw }}}}{\sqrt{Z_{\text {siw }}}-\sqrt{Z_{o}}}
\end{array}\right.
$$

The design of the presented transition is split into two steps; in A band width of nearly 2.5 $\mathrm{GHz}$ is achieved the first step, an empty substrate integrated waveguide is designed, its impedance is calculated using the closed Equation (2). A $50 \Omega$ microstrip line width is also calculated in this step. In the second step, Equation (4) allows to calculate $\mathrm{Z}_{1}$, then the width $\mathrm{W}_{2}$ is determined [9]. Later, Equation 2-b gives the length $\mathrm{L}$ which corresponds to the minimum return loss. An additional step (if necessary), is to use a full-wave simulator as HFSS in order to refine the values of $Z_{1}$ and $L_{1}$.

$$
Z_{1}=\frac{120 \pi}{\sqrt{\varepsilon_{e f f}}\left(\left(\frac{w_{1}}{h}\right)+1.393+0.667 \cdot \ln \left(\frac{w 1}{h}+1.444\right)\right)}
$$




\section{Simulation Results}

Table 1 recapitulates values of three SIW structures versus frequency, geometrical parameters are calculated using the presented procedure and compared with values obtained using HFSS software. We can see that this method gives a quick solution and, at least an initial optimum point for sizing the transition. These obtained calculations of dimensions $(\mathrm{L} \times \mathrm{W})$ can be taken into account as a first optimization. Simulation software can quickly obtain better optimization.

TABLE I. GeOMETRICAL PARAMETER VALUES IN Millimiter.

\begin{tabular}{|c|c|c|c|c|c|c|}
\hline \multirow{2}{*}{ Parameters } & \multicolumn{2}{|c|}{$\begin{array}{c}\text { Optimum value } \\
\text { using HFSS }\end{array}$} & \multicolumn{2}{c|}{$\begin{array}{c}\text { Calculated } \\
\text { parameters }\end{array}$} & \multicolumn{2}{c|}{ Relative error \% } \\
\cline { 2 - 7 } & $\boldsymbol{W}_{\boldsymbol{I}}$ & $\boldsymbol{L}_{\boldsymbol{1}}$ & $\boldsymbol{W}_{\boldsymbol{I}}$ & $\boldsymbol{L}_{\boldsymbol{I}}$ & $\boldsymbol{W}_{\boldsymbol{1}}$ & $\boldsymbol{L}_{\boldsymbol{1}}$ \\
\hline $\begin{array}{c}\mathrm{f}_{0}=10 \mathrm{GHz} \\
\varepsilon \mathrm{r}=4.4\end{array}$ & 1.8121 & 3.9105 & 1.98510 & 3.5730 & 8.7149 & 8.6306 \\
\hline $\begin{array}{c}\mathrm{f}_{0}=14.5 \mathrm{GHz} \\
\varepsilon \mathrm{r}=2.2\end{array}$ & 2.4152 & 3.8221 & 2.2214 & 3.3368 & 8.0242 & 12.6972 \\
\hline $\begin{array}{c}\mathrm{f}_{0}=21 \mathrm{GHz} \\
\varepsilon \mathrm{r}=3.55\end{array}$ & 1.3945 & 2.1572 & 1.2204 & 1.8082 & 12.4848 & 16.1784 \\
\hline
\end{tabular}

The presented design procedure has been used in order to design transition for a substrate integrated waveguide working at $10 \mathrm{GHz}$ with FR4-epoxy substrate, $\varepsilon_{\mathrm{r}}=4.4$. The impedance of the transition is $Z_{1}=36.2307 \Omega$, which corresponds to a width of $1.9810 \mathrm{~mm}$.

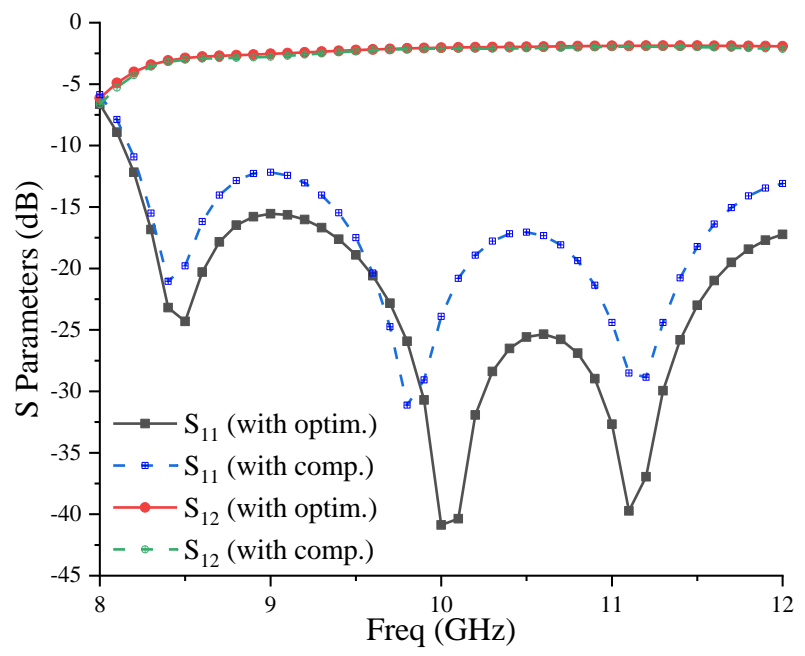

Fig. 3.Scattering parameters of SIW with deduced and HFSS optimized $(\mathrm{L} \times \mathrm{W})$ transition dimension for $\mathrm{f}=10 \mathrm{GHz}$. 
Comparisons between the $\mathrm{S}$ parameters of the first structure for our calculated values $(\mathrm{L} \times \mathrm{W})$ and those optimized by HFSS of the 3 different frequency values are presented in Figs. 3, 4 and 5, we can note:

1- A bandwidth of around $2.5 \mathrm{GHz}$ is reached for the first range of frequency (Fig.3).

2- A good agreement is obtained between the calculated and optimized $(\mathrm{L} \times \mathrm{W})$ of $\mathrm{S}$ parameters.

3 - For high-frequency values our optimal values of $(\mathrm{L} \times \mathrm{W})$ give a better result of $\mathrm{S}$ parameter than that given by the optimal values obtained with HFSS (Figs.5 and 6).

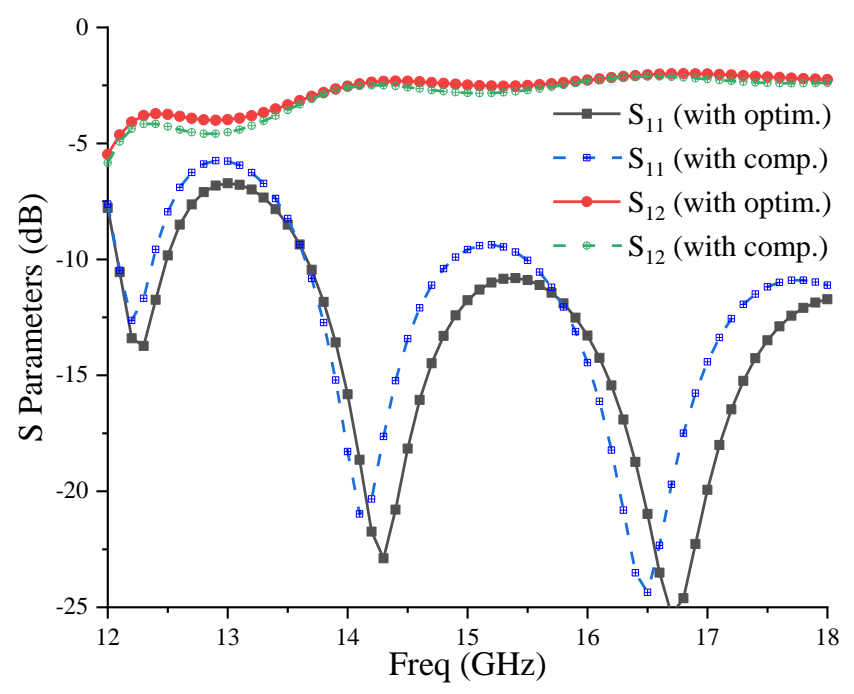

Fig. 4.Scattering parameters of SIW with deduced and HFSS optimized $(\mathrm{L} \times \mathrm{W})$ transition dimension for $\mathrm{f}=14.5 \mathrm{GHz}$.

Now we turn our attention to the directional coupler, where the design method presented below is used to set, this time, a microstrip-to-directional coupler transition. The directional coupler is a cruciform $\mathrm{H}$-plane $3 \mathrm{~dB} / 90^{\circ}$ hybrid coupler that consists of a rectangular cavity connected to four input/output ports. Metallic vias are inserted within the crossing area in order to produce the desired directional properties [10-13], Figure 6 shows the structure of the considered coupler. 


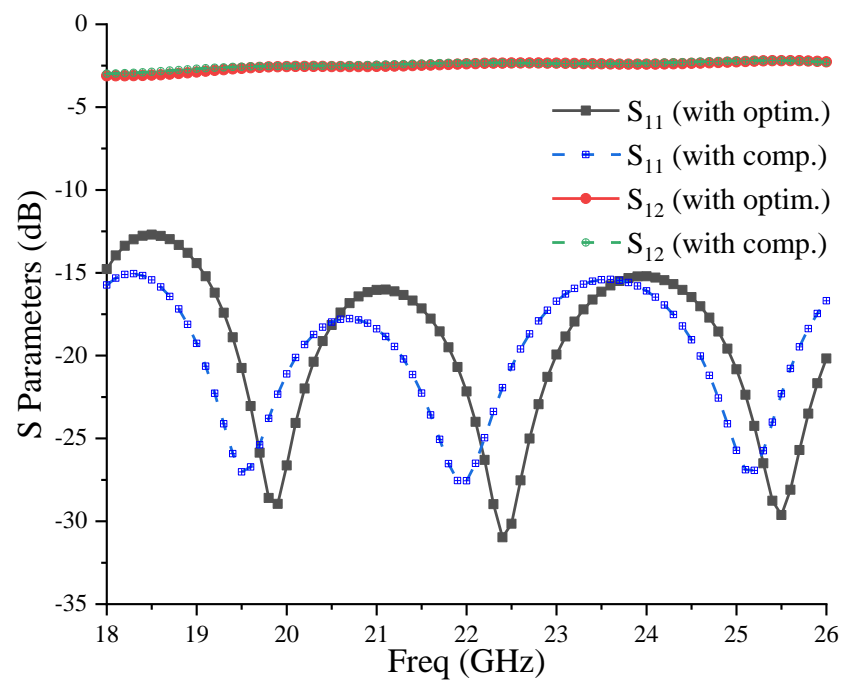

Fig. 5.Scattering parameters of SIW with deduced and HFSS optimized $(\mathrm{L} \times \mathrm{W})$ transition dimension for $\mathrm{f}=21 \mathrm{GHz}$.

In this section, we won't present the coupler design method, but we only focus on the design of transition using the method presented below. A directional coupler is a network of four impedances that can be used as a power divider or power combiner by using an even-odd mode analysis technique and symmetric across the mid-plane characteristic. The coupler can be decomposed to a simple circuit as presented in Figure 7. The input impedance at any port of the coupler can be expressed by Equation 5 . In this case $\mathrm{Z}_{\mathrm{siw}}=\mathrm{Z}_{\mathrm{o}}[9]$.

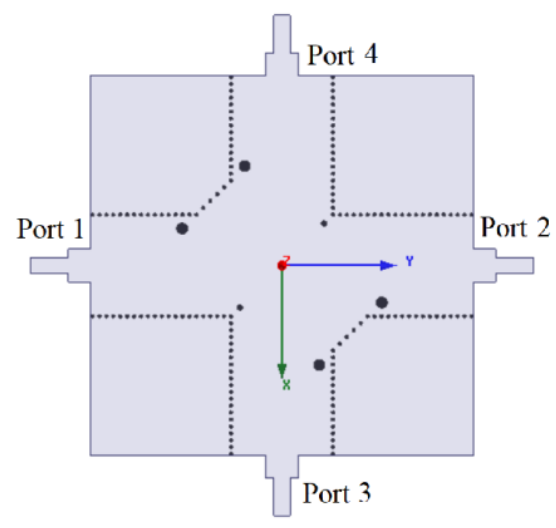

Fig. 6.Cruciform $\mathrm{H}$-plane $3 \mathrm{~dB} / 90^{\circ}$ hybrid coupler

The design strategy is divided into two parts; the first part consists in optimizing the coupler without transition, where the geometrical parameters of the metallic via give the desired $\mathrm{S}$ parameters. In the second part; the transition will be added where the dimensions of this one 
will be calculated by the method presented below. Note that the impedance of the load is the impedance of the equivalent waveguide.

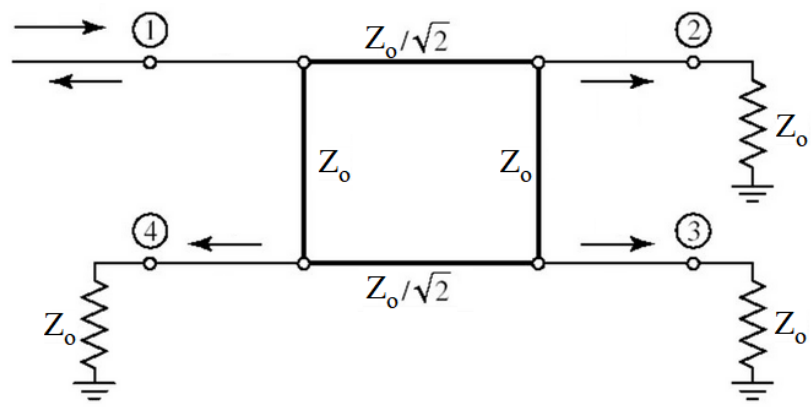

Fig. 7.Circuit of the branch-line hybrid coupler.

A $3 \mathrm{~dB}$ coupler operating on the X-band is designed. The substrate is Duroid 5870 with $\varepsilon_{\mathrm{r}}=$ 2.33 and $\mathrm{h}=0.787 \mathrm{~mm}$. the transition dimensions are optimized using HFSS simulator and using the proposed method. The optimized length and width are $6.63 \mathrm{~mm}$ and $4.76 \mathrm{~mm}$, respectively. The calculated length and width are $5.2 \mathrm{~mm}$ and $4.2 \mathrm{~mm}$, respectively. It can be seen that the calculated dimensions are close to the optimized dimensions. It should be noted that it is impossible to determine the correct dimensions using the presented method but it can be used to determine start values for optimization.

Figures 8 and 9 show the coupler S-parameters, we have a return loss lower than $-35 \mathrm{~dB}$ at the operating frequency.

According to Figure 8, a simple shift in the bandwidth is noticed, even the optimization of the HFSS is similar to our case for $\mathrm{S}_{11}$ and $\mathrm{S}_{13}$, whereas for $\mathrm{s}_{12}$ and $\mathrm{s}_{14}$, they are almost identical (Fig.9). 


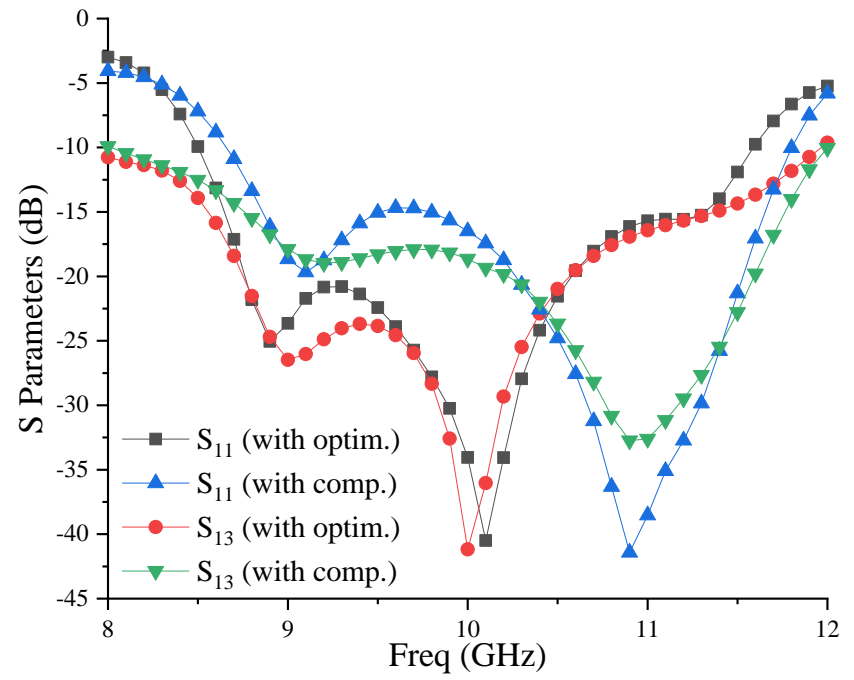

Fig. 8.Simulated $\mathrm{S}_{11}$ and $\mathrm{S}_{13}$ parameters of cruciform coupler with optimized and our computed (LxW)

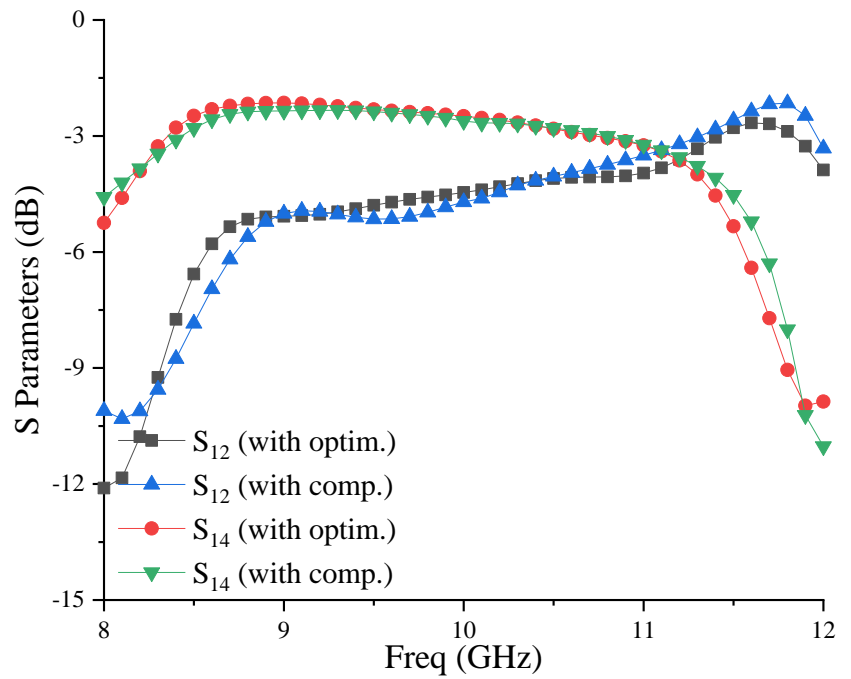

Fig. 9.Simulated $\mathrm{S}_{11}$ and $\mathrm{S}_{13}$ parameters of cruciform coupler with optimized and our computed (L×W).

\section{Conclusion}

In this work, analytical equations for the design of a discontinuity step transition are presented. The low reflection approximation method is used in order to find the relationship between all impedances and reflection factors. The proposed equations have been applied to 
design several transitions at different frequencies. In all cases, a good return loss has been obtained. The design procedure was applied for a microstrip-to-directional coupler transition. It allows finding a good starting point for optimization.

Acknowledgments. This work is funded by FCT/MCTES through national funds under the project UIDB/50008/2020-UIDP/50008/2020. This work is part of the POSITION-II project funded by the ECSEL joint Undertaking under grant number Ecsel- 345 7831132-Postitio-II2017-IA.

\section{References}

[1] M. Ando, J. Hirokawa and Al, "Novel single-layer waveguides for high-efficiency millimeter wave arrays", IEEE millimeter waves conference proceedings, pp.177-180, 1997.

[2] D. Deslandes and K. Wu, "Accurate modeling, wave mechanism, and design consideration of a substrate integrated waveguide," IEEE Trans. Microwave Theory \& Tech., vol. 54, no. 6,pp. 25162526, Jun. 2006.

[3] Th. R. Jones and M. Daneshmand, "Miniaturized Slotted Bandpass Filter Design Using a Ridged HalfMode Substrate Integrated Waveguide", IEEE Microwave and Wireless Components Letters, Vol. 26, no. 5, pp 343-346, MAY 2016

[4] L. Jie, Q. Cui and F. Lin, 'Reconfigurable HMSIW Quadrature Coupler ', IEEE Microwave and Wireless Components Letters, Vol.29, no: 10 , pp. 648-651 Oct. 2019.

[5] A. E. I. Lamminen, A. R. Vimpari, and J. Säily, "UC-EBG on LTCC for 60-GHz Frequency Band Antenna Applications ", IEEE Transactions on antennas and propagation, vol. 57, no. 10, pp. 29042912, october. 2009.

[6] K. Tekkouk, M. Ettorre and R. Sauleau, " Multibeam Pillbox Antenna Integrating Amplitude Comparison Monopulse Technique in the $24 \mathrm{GHz}$ Band for Tracking Applications', IEEE Transactions on Antennas and Propagation, Vol.66, no. 5 , pp. 2616 - 2621, May 2018.

[7] N. Guellil, F. Djahli, and C. Zebiri, "A new formula for the optimum width of Substrate Integrated Waveguide". Advanced Electromagnetics, 8(4), 39-43, 2019.

[8] E. Miralles, H. Esteban, C. Bachiller, A. Belenguer and Vicente E. Boria, "Improvement for the design equations for tapered Microstrip-to-Substrate Integrated Waveguide transitions", International Conference on Electromagnetics in Advanced Applications, Oct. 2011.

[9] D. M. Pozar, "Microwave Engineering", John Wiley \& Sons, Inc, 4th ed. USA, 2012.

[10] T. Djerafi and K. Wu, "Super-compact substrate integrated waveguide cruciform directional coupler," IEEE Microw.Wireless Compon. Lett., vol. 17, no.11, pp. 757-759, Nov. 2007.

[11]T. Djerafi, J. Gauthier, and K. Wu, "Quasi-optical cruciform substrate integrated waveguide (SIW) coupler for millimeter-wave systems," International Microwave Symposium Digest (MTT), pp 1-4, 2010.

[12]Elfergani, I.T.E.; Hussaini, A.S.; Rodriguez, J.; Abd-Alhameed, R. Antenna Fundamentals for Legacy Mobile Applications and Beyond; Springer: Cham, Switzerland, 2017; pp. 1-659.

[13] Issa Elfergani, Amjad Iqbal, Chemseddine Zebiri, Abdul Basir, Jonathan Rodriguez, Maryam Sajedin, Artur De Oliveira Pereira, Widad Mshwat, Raed Abd-Alhameed, Sadiq Ullah. Low-Profile and Closely Spaced Four-Element MIMO Antenna for Wireless Body Area Networks. Electronics. 2020; $9(2): 258$. 